\title{
Pulsed Phase Thermography applied on complex structures: modelling and numerical analysis
}

\author{
By F.L. Rodríguez ${ }^{\star}$,V.P. Nicolau*, and X. Maldague**
}

*Dept. of Mechanical Engineering, Universidade Federal de Santa Catarina, 88040-900,Florianópolis, Santa Catarina, Brazil, flopez@cwpanama.net

**Electrical and Computing Engineering Dept., Université Laval, Quebec City (Quebec), Canada G1K 7P4, xavier.maldague@gel.ulaval.ca

\begin{abstract}
This main objective of this work is to develop and solve a thermal-numerical model to study the application of Pulsed-Phase Thermography to the nondestructive inspection of complex structures. To do this aim, it is proposed a mathematical model based on the 3D transient energy equation, in order to predict the temperature field when applied a thermal excitation to the specimen under inspection. The proposed thermal model takes into consideration several aspects that affect the heat diffusion through the material, such as the anisotropy of the material, the heat transfer between the surface of the specimen and the external environment and the heat generation by the absorption of electromagnetic radiation. The numerical solution was developed using the Finite Volume Method (FVM), in which the approximated equations are obtained by performing energy balances for each elementary volumes. It is analysed the thermal response of the specimen in time and frequency domain once is applied the Fourier Transform to the thermogram sequence obtained by numerical simulation.
\end{abstract}

\section{Introduction}

Infrared and Thermal Methods rely on the measurement and the subsequent analysis of the emitted thermal energy (for instance, infrared radiation), to predict and evaluate the presence of anomalies within the material. The techniques within this category are known as Thermal Nondestructive Testing (TNDT) methods, and include several measurement techniques which depend primarily on the wavelength associated to the radiation emitted by the object under study and also on the temporal and spatial resolution of the equipments. Among the different TNDT techniques, Infrared Thermography (IRT) has become one of the most attractive methods for defect detection and characterization. IRT as a NDT is a non-contact and real-time sensing method that provided the surface temperature through the measurement of the infrared radiation emitted by the surface. When the heat flux within the material is altered by the presence of defects or any anomalies, alterations or contrasts in the temperature field appear in the surface. The application of IRT to detect subsurface defects is mainly based on the acquisition and analysis of those thermal patterns, known as thermograms [1].

The use of IRT as a NDT requires the application of an external stimulation in order to provoke a heat flux within the material. This approach is known as Active Thermography Approach. Several excitations methods are available, being optical/thermal and mechanical excitation the most widely used nowadays. This work is based on the optical/thermal methods, specifically Pulsed-Phase Thermography. This inspection method is deployed in transient regime and consists in transferring heat by radiation (for instance, using a thermal pulse) to the surface of the material while the thermal response is monitored using an infrared camera. The resulting temperature evolution field is comprised of internal reflections between the interfaces of subsurface defects and the surface of each wave launched to the specimen, based on the frequency spectrum available in the stimulation pulse.

However, in order to properly conduct the inspection by IRT - as well as for an adequate interpretation of the results - several parameters and physical process must be understood. Those parameters and physical process may vary from one case to another, and mostly depend on the structure of the material and characteristic associated to the defects being analysed. In this sense, numerical simulation constitutes a valuable tool to study the different phenomena that take places during an inspection by infrared thermography, thus enabling to analyze the effectiveness of the inspection method and the optimization of real tests.

Investigations concerned to the computational simulation of infrared thermography inspections using different kind of excitations modes have grown over the last few years (it can be found in reference [2] a brief summary of various simplifications method to describe the heat transfer phenomena during an inspection by IRT). This fact has allowed the application and improvement of the IRT techniques to situation to which until recently, was not possible to inspect, such as materials with high thermal conductivity and complex structures, such as presented in this paper.

In this work is studied the application of Pulsed Phase Thermography to the inspection of complex structures, through the modelling and numerical simulation of the heat transport phenomena involved in the TNDT. For this purpose, it is proposed a thermal-numerical model based on the 3D transient energy equation to describe the thermal behaviour of the specimen when a thermal pulsed is uniformly applied to its surface. The model takes into consideration the heat transfer by convection and radiation between all the specimen surfaces and the environment. Also, the anisotropy of the 
composite material is considered. The defects under consideration are delaminations between the carbon-fiber plies and between the composite/metal interface. The numerical solution is implemented using the Finite Volume Method, which consists in integrating over an elementary volume, on space and time, the energy equation in the conservative form. It is analysed the thermal response of the specimen due to the application of the thermal pulse in the time and frequency domain, after applying the Fourier Transform to the simulated thermogram sequence.

\section{Proposed Thermal Model}

The proposed thermal model is depicted in figure 1. The complex structure is a metal/composite joint having different kind of defects at the interface of both materials. The interfacial defects (such as delimitations and poor adhesion) have different size and depth. The composite material is modelled as a porous media and the thermophysical properties of each phase (matrix and fibbers) are calculated based on the local volume averaging [3]. The specimen is in thermal equilibrium at the beginning of the IR inspection $\left(t=t_{o}\right)$; at time $\left(t=t_{1}\right)$ an external stimulation by thermal radiation is applied to the surface, causing the propagation of a thermal front through the material by diffusion, and to transfer heat by convection and radiation between its surface and the external environment. The resulting thermal response of the material is monitored via infrared thermal emission, as is showed in figure 1(a):

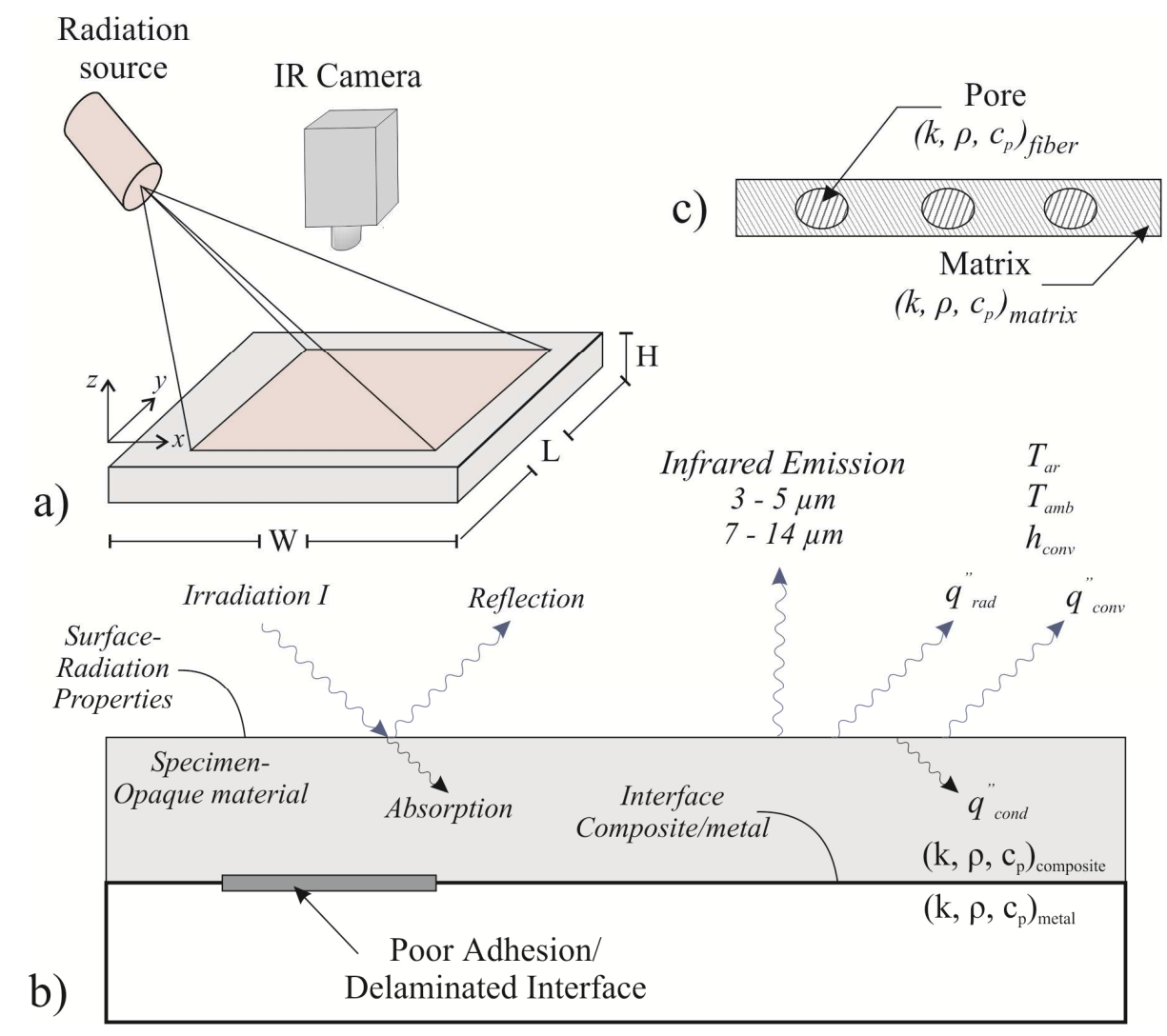

Fig. 1. Proposed thermal model to study the inspection by PPT in bonded components. (a) Physical domain under study. (b) Heat fluxes participating during the TNDT and their interaction with the environment and internal interfaces. (c) Schematic representation of the composite material.

The governing equations that describe the heat diffusion process when a pulsed thermal excitation is applied at the surface of the material are:

$$
\begin{aligned}
& \left(\rho c_{p}\right)_{\text {metal }} \frac{\partial T_{\text {metal }}}{\partial t}=\nabla \cdot\left(k_{\text {metal }} \cdot \nabla T_{\text {metal }}\right)+q^{\prime \prime \prime} \\
& \left(\rho c_{p}\right)_{\text {comp }} \frac{\partial T_{\text {comp }}}{\partial t}=\nabla \cdot\left(k_{\text {comp }} \cdot \nabla T_{\text {comp }}\right)+q^{\prime \prime \prime}
\end{aligned}
$$


The initial condition is given by the Eq. (3):

$$
T\left(x, y, z, t_{o}\right)=T_{a m b}
$$

and the boundary conditions, including the heat transfer by convection and radiation between all the surfaces and the environment are defined by:

$$
n \cdot(k \cdot \nabla T)=h_{\text {conv }}\left(T_{a i r}-T\right)+\sigma \varepsilon\left(T_{a m b}^{4}-T^{4}\right)
$$

fluxes,

Also, boundary conditions at the interfaces of the materials are given by the continuity of temperature and heat

$$
\begin{aligned}
& T_{\text {metal }}=T_{\text {comp }} \\
& n \cdot\left(k_{\text {comp }} \cdot \nabla T_{\text {comp }}\right)=n \cdot\left(k_{\text {metal }} \cdot \nabla T_{\text {metal }}\right)
\end{aligned}
$$

In Eqs. (1) and (2), the subscript "metal" and "comp" represents each of the mediumspresent in the bonded structure (metallic and composite), which are considered to be steel and carbon fibber reinforced polymer, respectively. Given that the composite medium is anisotropic, it is necessary to develop an appropriate formulation that takes into consideration the effective thermophysical properties. Based on the rule-of-mixture, the effective heat capacity of the composite medium is estimated by the weighted average of the specific properties of each phase involving the volume fraction [3]:

$$
\left(\rho c_{p}\right)_{c o m p}=\phi_{f}\left(\rho c_{p}\right)_{f}+\left(1-\phi_{f}\right)\left(\rho c_{p}\right)_{m}
$$

where $\phi_{f}$ denotes the volume fraction of the fibber, given by:

$$
\phi_{f}=\frac{V_{f}}{V_{T}}=\frac{V_{f}}{V_{f}+V_{m}}
$$

and the subscripts $f$ and $m$ denote the carbon fiber and epoxy resin matrix [see figure 1(c)].The effective thermal conductivity is calculated considering two different thermal conductivities: thermal conductivity parallel and transversal to the $x-y$ plane, which are determined as a function of each of the constituents of the composite medium [4]:

$$
\begin{aligned}
& k_{p}=k_{f} \phi_{f}+k_{m}\left(1-\phi_{f}\right) \\
& \frac{1}{k_{t}}=\frac{\phi_{f}}{k_{f}}+\frac{\phi_{m}}{k_{m}}
\end{aligned}
$$

Where $k_{f}$ and $k_{m}$ are the fiber and epoxy resin thermal conductivities, respectively.

The source term $q^{\prime \prime \prime}$ appearing in Eqs. (1) and (2) is related to the heat generation within the material due to absorption of electromagnetic radiation; this term accounts the portion of the radiation that is absorbed by the material (absortivity) and the optical penetration coefficient (distance over which the incident radiation decays to $1 / e$ of its surface). Thus, from electromagnetic theory, the volumetric heat generation by absorption of radiation can be written as:

$$
q^{\prime \prime \prime}=\frac{I \cdot \delta(t) \cdot[1-\rho] \cdot \exp (-a \cdot z)}{z}
$$

In Eq. (10), $I$ represents the intensity of the thermal applied excitation, $\delta(t)$ is the duration of the thermal pulse in $\mathrm{ms},(1-\rho)$ corresponds to the portion of radiation absorbed by the surface of the material and $a$ is the optical absorption coefficient of the medium, which depends on the extinction coefficient and is a spectral dependent quantity. 


\subsection{Numerical solution approach}

The solution of the governing equations is carried out using the Finite Volume Method (FMV), which has been successfully used to investigate different parameters during the deployment of a thermal nondestructive testing by infrared thermography [5]. This method consists of the integration of space and time, over an elemental volume, the energy equation in the conservative form, which is equivalent to performing energy balances over all the elemental volumes. The domain under study was divided into twenty-seven (27) types of elemental volume, according to the position and the heat transferred to the external environment and its neighbourhoods. The energy balances provides the local temperature equation at the center of the volume (cell-center mesh), while the material thermal properties (thermal conductivity and specific heat) remain stored at the interface of the control volume. Thus, integrating space and time over an elemental volume, is obtained discrete energy balance for internal volumes, given by:

$$
\begin{aligned}
& \frac{M_{p} T_{p}-M_{p}^{o} T_{p}^{o}}{\Delta t}=\left[\left.\frac{k}{c_{p}} \frac{\partial T}{\partial x}\right|_{e} ^{\theta}-\left.\frac{k}{c_{p}} \frac{\partial T}{\partial x}\right|_{w} ^{\theta}\right]^{\theta} \Delta y \Delta z+ \\
& {\left[\left.\frac{k}{c_{p}} \frac{\partial T}{\partial y}\right|_{n} ^{\theta}-\left.\frac{k}{c_{p}} \frac{\partial T}{\partial y}\right|_{s} ^{\theta}\right]_{s} \Delta x \Delta z+} \\
& {\left[\left.\frac{k}{c_{p}} \frac{\partial T}{\partial z}\right|_{b} ^{\theta}-\left.\frac{k}{c_{p}} \frac{\partial T}{\partial z}\right|_{f} ^{\theta}\right]_{\Delta x \Delta y}}
\end{aligned}
$$

Applying the same methodology at the external surfaces [see Eq. (4)], results in the energy balance for the surfaces of the sample test:

$$
\begin{aligned}
& \frac{k}{\Delta x / 2}\left(T_{(i+1, j, k)}-T_{(i, j, k)}\right)=\sigma \cdot \varepsilon\left(T_{(i, j, k)}^{4}-T_{a m b}^{4}\right) \Delta y \Delta z+ \\
& h_{c o n v} \cdot\left(T_{(i, j, k)}-T_{a i r}\right) \Delta y \Delta z
\end{aligned}
$$

In Eq. (12), the first term corresponds to the transient (or thermal inertia) term, followed by the incoming and outgoing conduction fluxes in each of the spatial coordinates. Due to the diffusive nature of the internal heat conduction process within the sample test, the derivatives present in the discrete energy balance were approximated using central difference scheme. Likewise, the implicit formulation was used as a temporal interpolation function.

\subsection{Convection heat transfer coefficients}

As shown in Eq. (13), the natural convection heat transfer coefficient must be calculated in order to quantify the amount of thermal energy transferred to the external environment by the sample surfaces. In this work the convection heat transfer coefficients are calculated as proposed by [6], taking into consideration the position of the surfaces and the temperature of the fluid film. Thus, for the lateral surfaces of the sample (4 surfaces), the convection coefficient $\boldsymbol{h}_{\text {lat }}$ is given by Eq. (14):

$$
h_{\text {lat }}=\frac{k}{H} \overline{N u}_{L}
$$

In Eq. (12), $\overline{N u}_{L}$ is the Nusselt number based on $L$, which is obtained by the expression recommended by Churchill and Chu [7], and $k$ represents the thermal conductivity of the fluid film. For the upper surface of the sample, the convection coefficient $h_{u p p}$ is defined by Eq. (15):

$$
h_{u p p}=0.15 \frac{k}{A_{s} / P} R a_{L}^{1 / 3}
$$


Finally, for the lower surface the convection coefficient $h_{\text {low }}$ is calculated by Eq. (16):

$$
h_{\text {low }}=0.27 \frac{k}{A_{s} / P} R a_{L}^{1 / 4}
$$

In Eqs. (15) and (16), the term $A_{s} / P$ represents the characteristic length defined as the relation between the area and the perimeter of the surface under consideration and $R a_{L}$ is the Raleigh number based on $L$, which is determined as proposed by [6].

\section{Numerical simulation results}

In order to calculate the temperature field within the material and its surface, it is necessary to solve the set of energy balance equations developed in Section 2.1. Because of the implicit formulation used as a temporal interpolation function, these set of discrete equations were solved using the Gauss-Seidel iterative method. A computational program called PulsedThermography ${ }^{\circledR}$ was developed to compute and analyse the thermal response of the material when an optical/thermal excitation is applied to the sample surface. The program was developed using the MatLab ${ }^{\circledR}$ platform [8] and it provides the surface thermal map of the material, in a similar way as in a real NDT by infrared thermography. This way, several parameters can be studied, such as the adequate amount of thermal energy (intensity and duration of the thermal pulse) that must be delivered to reveal defects located at different depths, thermophysical properties of the material under inspection, and the influence of the external environment under which the inspection is carried out.

Table 1 presents the parameters and thermophysical properties used in the numerical simulation. All numerical results were obtained using the same mesh and time step, assuring then the consistency of the results.

Table 1. Parameters and thermal properties used in the numerical simulation

\begin{tabular}{|c|c|c|c|}
\hline Symbol & $\begin{array}{l}\text { Parameters and thermophysical } \\
\text { properties }\end{array}$ & Value & Units \\
\hline$n_{x} \times n_{y} \times n_{z}$ & number of volumes & $60 \times 60 \times 50$ & volumes \\
\hline$d_{x} \times d_{y} \times d_{z}$ & dimension of the volumes & $5.2 \times 5.2 \times 0.42$ & $\mathrm{~mm}$ \\
\hline$\Delta t$ & time step & $10 e^{-3}$ & $\mathrm{~s}$ \\
\hline$T_{0}$ & initial temperature & 293.15 & $\mathrm{~K}$ \\
\hline$T_{a m b}$ & ambient temperature & 293.15 & $\mathrm{~K}$ \\
\hline$T_{\text {air }}$ & air temperature & 293.15 & $\mathrm{~K}$ \\
\hline$H \times L X W$ & sample dimensions & $0.3 \times 0.3 \times 0.002$ & $\mathrm{~m} \times \mathrm{m} \times \mathrm{m}$ \\
\hline$\Phi_{\text {fiber }}$ & volumetric fraction & 0.5 & - \\
\hline$\rho_{\text {fiber }}$ & density(fiber) [8] & 1750 & $\mathrm{~kg} / \mathrm{m}^{3}$, \\
\hline$c p_{\text {fiber }}$ & specific heat (fiber) [8] & 710 & $\mathrm{~J} / \mathrm{kg}-\mathrm{K}$ \\
\hline$k_{\text {fiber }}$ & thermal conductivity (fiber) [8] & 50 & $\mathrm{~W} / \mathrm{m}-\mathrm{K}$ \\
\hline$\rho_{\text {matrix }}$ & Density (epoxy) [8] & 1370 & $\mathrm{~kg} / \mathrm{m}^{3}$ \\
\hline$c p_{\text {matrix }}$ & specific heat (epoxy) [8] & 1884 & $\mathrm{~J} / \mathrm{kg}-\mathrm{K}$ \\
\hline$k_{\text {matrix }}$ & thermal conductivity (epoxy) [9] & 0.3 & $\mathrm{~W} / \mathrm{m}-\mathrm{K}$ \\
\hline$\rho_{\text {metal }}$ & density (steel 304) [9] & 8000 & $\mathrm{~kg} / \mathrm{m}^{3}$ \\
\hline$c p_{\text {metal }}$ & specific heat (steel 304) [9] & 500 & $\mathrm{~J} / \mathrm{kg}-\mathrm{K}$ \\
\hline$k_{\text {metal }}$ & thermal conductivity (steel 304) [9] & 16.2 & $\mathrm{~W} / \mathrm{m}-\mathrm{K}$ \\
\hline$\rho_{\text {comp }}$ & density (effective) & 1560 & $\mathrm{~kg} / \mathrm{m}^{3}$ \\
\hline$c p_{\text {comp }}$ & specific heat (effective) & 1297 & $\mathrm{~J} / \mathrm{kg}-\mathrm{K}$ \\
\hline$k_{\text {parallel }}$ & thermal conductivity (parallel) & 25.15 & $\mathrm{~W} / \mathrm{m}-\mathrm{K}$ \\
\hline$k_{\text {transveral }}$ & thermal conductivity (transversal) & 0.60 & $\mathrm{~W} / \mathrm{m}-\mathrm{K}$ \\
\hline$\rho_{\text {defect }}$ & density (Teflon) [10] & 2200 & $\mathrm{~kg} / \mathrm{m}^{3}$ \\
\hline$c p_{\text {defect }}$ & specific heat (Teflon) [10] & 1040 & $\mathrm{~J} / \mathrm{kg}-\mathrm{K}$ \\
\hline$k_{\text {defect }}$ & thermal conductivity (Teflon) [10] & 0.23 & $\mathrm{~W} / \mathrm{m}-\mathrm{K}$ \\
\hline$h_{\text {upp }}$ & conv. coefficient upper surface & 4.74 & $\mathrm{~W} / \mathrm{m}^{2}-\mathrm{K}$ \\
\hline$h_{\text {low }}$ & conv. coefficient lower surface & 2.75 & $\mathrm{~W} / \mathrm{m}^{2}-\mathrm{K}$ \\
\hline$h_{\text {lat }}$ & conv. coefficient lateral surface & 8.21 & $\mathrm{~W} / \mathrm{m}^{2}-\mathrm{K}$ \\
\hline$P$ & power of the heat source & 10000 & W \\
\hline$\delta(t)$ & duration of the thermal pulse & 100,400 & $\mathrm{~ms}$ \\
\hline$\varepsilon$ & emissivity & 0.85 & - \\
\hline$\rho$ & reflectivity & 0.15 & - \\
\hline$a$ & absorption coefficient & $1.5071 \mathrm{e} 6$ & $\mathrm{~cm}^{-1}$ \\
\hline
\end{tabular}


The geometry and distribution of the defects within the specimen under consideration is depicted in figure 2 . The specimen contains defects with different sizes and depths. Defects located between the interfaces of each plies are located at $0.20,0.40$ and $0.60 \mathrm{~mm}$ from the surface. Its lateral size range from $3 \mathrm{~mm}$ to $15 \mathrm{~mm}$, all having square form. The defects located between the composite/metal interfaces are located at $0.80 \mathrm{~mm}$ depth and their lateral sizes are 20 $\mathrm{mm}$ and $25 \mathrm{~mm}$. The thermophysical properties of the defects are considered to be the same as Teflon (see table 1).

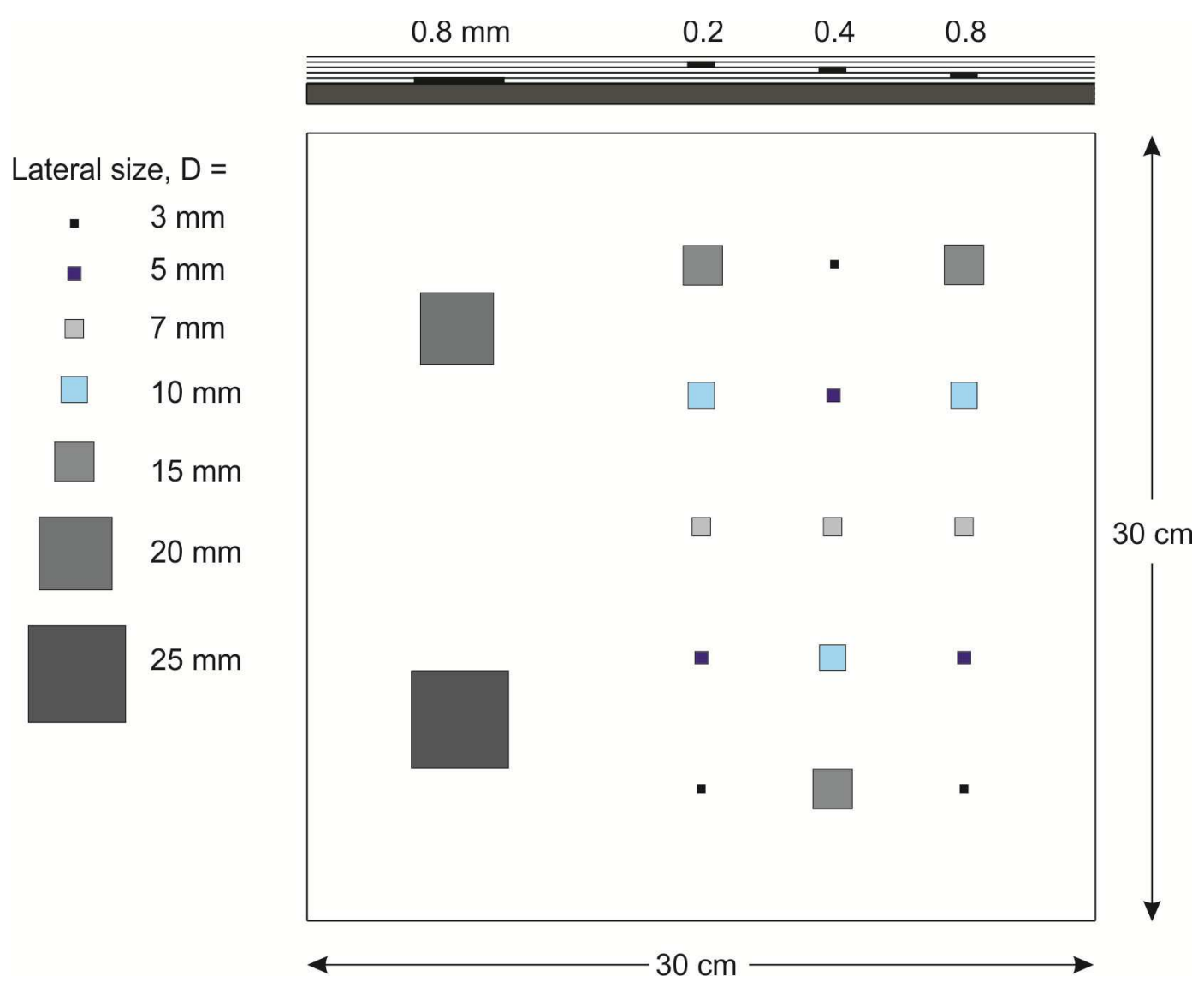

Fig. 2. Geometry of the sample test and configuration of the defects.

Figures $3(\mathrm{a})$ and $4(\mathrm{~b})$ shows the temperature evolution curves at different depths, considering a thermal pulse of $\mathrm{P}=$ $10000 \mathrm{~W}$ and duration of $100 \mathrm{~ms}$ and $400 \mathrm{~ms}$, respectively. This solution agrees the physical behaviour of the heat transfer process at the interior of the sample, which corresponds to a purely diffusive process. The difference between the temperature evolution curves at the surface and at the interior of the sample $(0.20,0.50$ and $2 \mathrm{~mm})$ denotes the heat transfer process by conduction; the higher the difference between those curves, the higher the amount of heat transferred by diffusion.

Taking into consideration the above mentioned, it is shown that in order to propagate the thermal front deeper, it is required to launch to the specimen a large amount of thermal energy. This fact directly affects the detection of deeper defects in the time domain, since it will enable the interaction of internal interfaces with the thermal energy being propagated. In the same way, the duration of the thermal pulse dominates the transient regime of the cooling process. As illustrated in figures 3 , the thermal equilibrium (within the material) when applied a thermal pulse with 100 ms of duration is reached after $5 \mathrm{~s}$ from the beginning of the inspection; meanwhile, when the duration is $400 \mathrm{~ms}$, the thermal equilibrium is reached after $6 \mathrm{~s}$. 


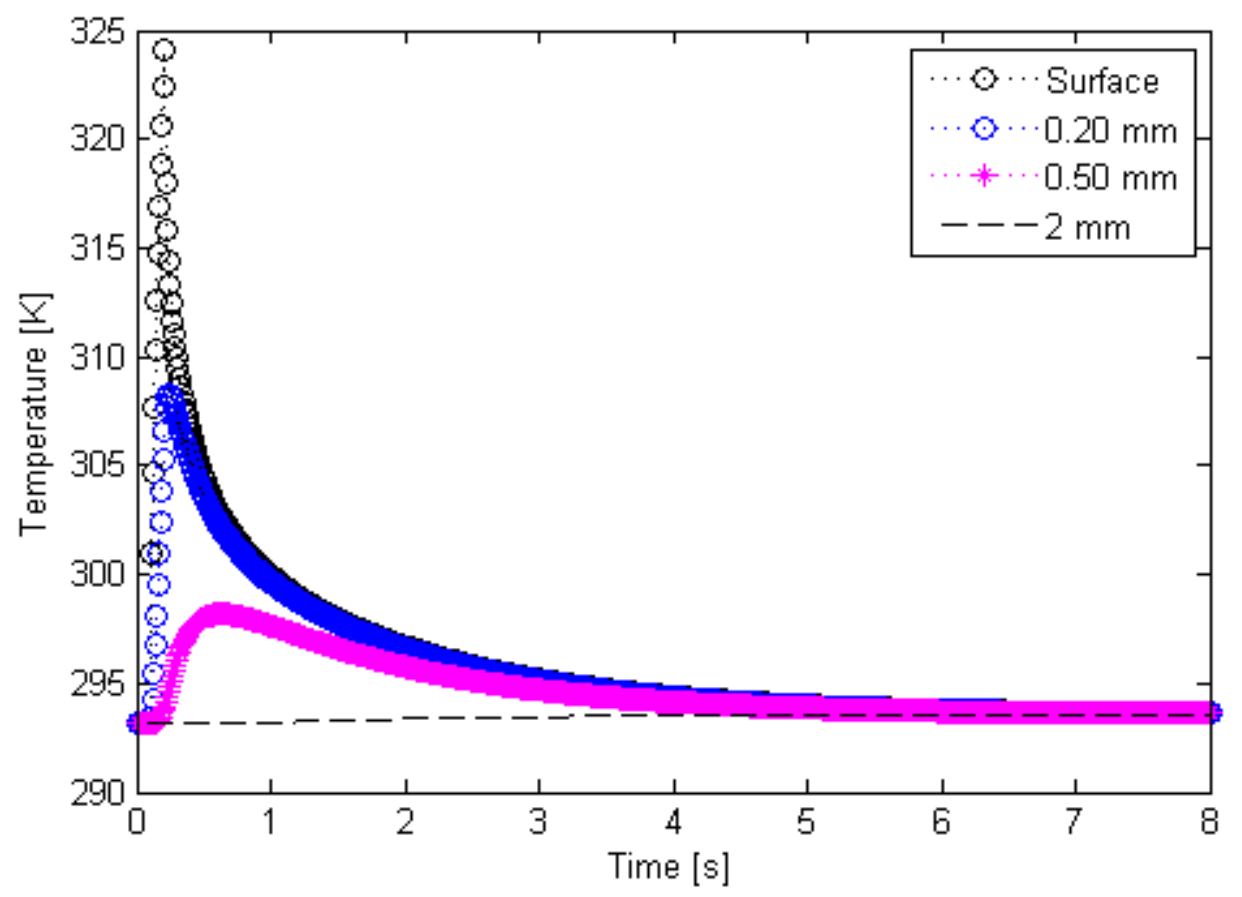

(a)

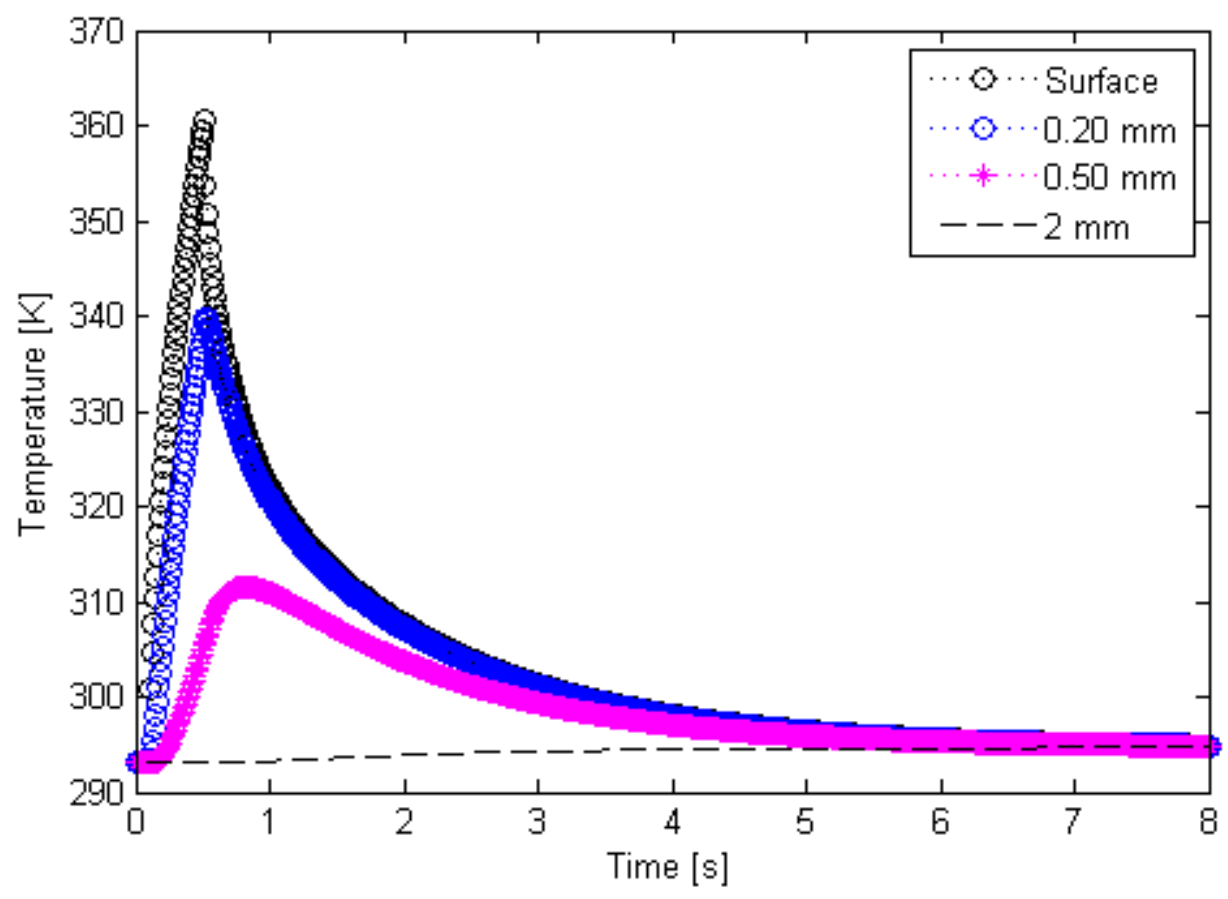

(b)

Fig. 3. Temperature evolution curves as a function of depth, considering a thermal pulse with $P=10000 \mathrm{~W}$ and duration of (a) $100 \mathrm{~ms}$ and (b) $400 \mathrm{~ms}$.

Figure 4 shows a thermal map sequence obtained by numerical simulation. The acquisition parameters adopted for those results were $\Delta t=100 \mathrm{frame} / \mathrm{s}$ and $w(t)=8 \mathrm{~s}$. At first observation, it can be noted that defects located at $0.2 \mathrm{~mm}$ can be detected $0.50 \mathrm{~s}$ after the application of the thermal pulse (figure $3 \mathrm{a}$ ), followed by the defects located at $0.40 \mathrm{~mm}$ (figure $3 \mathrm{~b}$ ) and $0.60 \mathrm{~mm}$ (figure $3 \mathrm{c}$ ). The defects at the interface of the composite and metallic materials, 
located both at $0.80 \mathrm{~mm}$ can be clearly detected $3.0 \mathrm{~s}$ after the beginning of the cooling process. These results confirm the scale presented by [11], which states that the observation time is function of the square of the depth, while the loss of thermal contrast is proportional to the cube of the depth. Consequently, deeper defects require more observation time to be detected and with a reduced thermal contrast.

It should also be noted that defectives zones present higher temperature than the sane areas. This is a consequence of the resistive effect of the defects that alter the heat flux applied on the surface, causing an increase in temperature due to the accumulation of heat on that region of the material. Using these heat transfer considerations, important key-features of the defects can be obtained and it is possible to determine what kind of defects are present in the material.

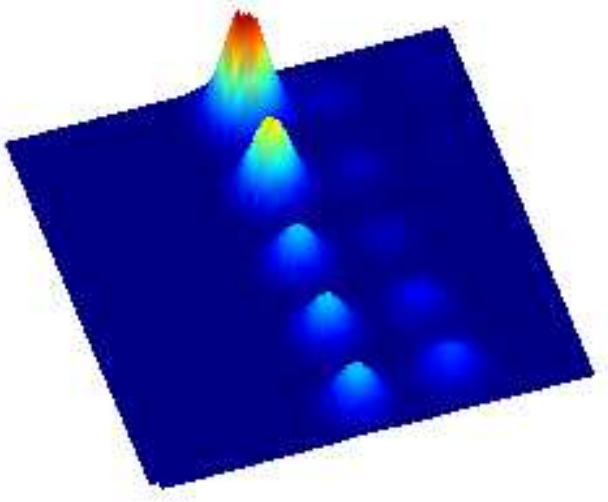

(a)

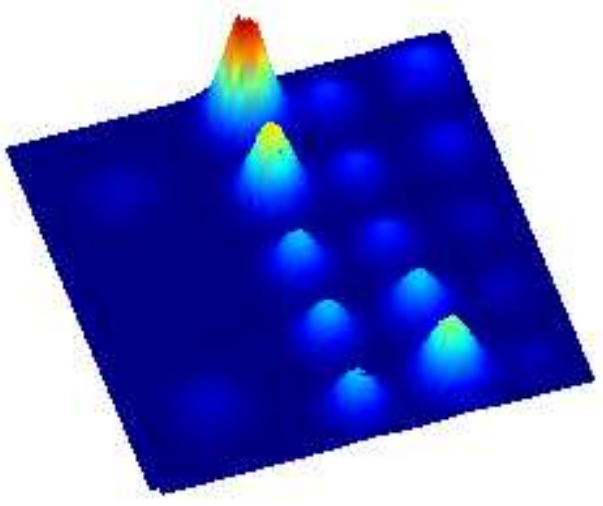

(c)
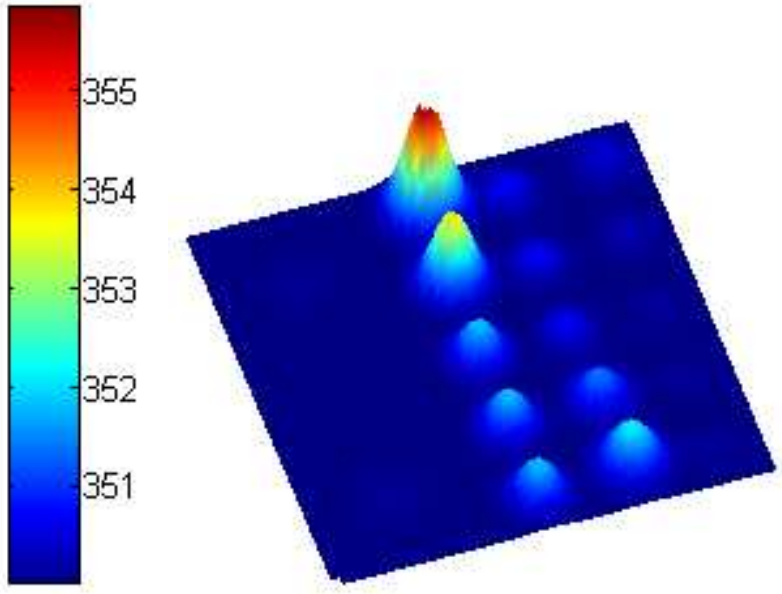

(b)
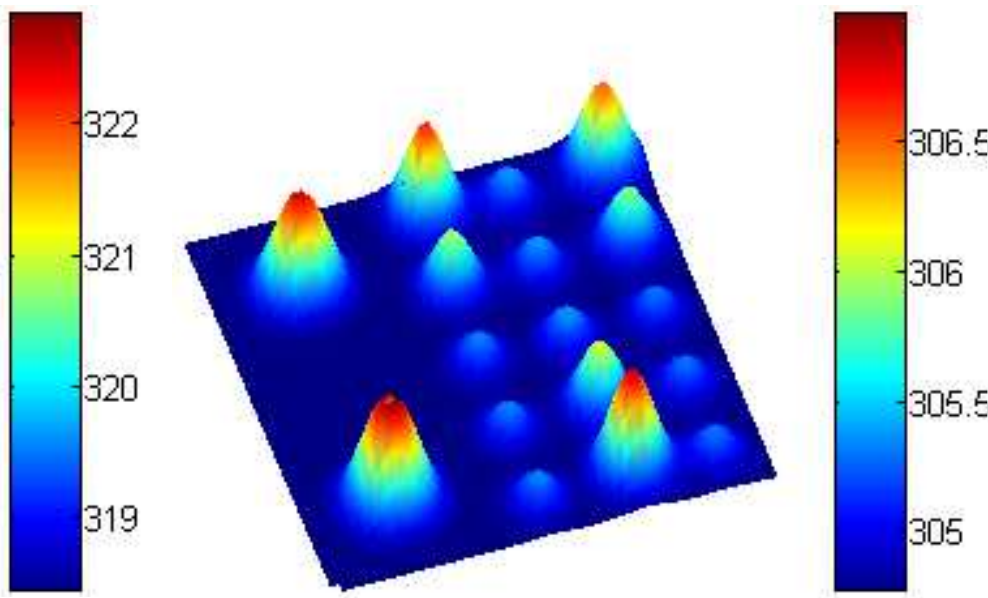

(d)

Fig. 3. Temperature evolution field of the surface obtained by numerical simulation. The thermal images correspond to: (a) $0.50 \mathrm{~s}$, (b) $0.70 \mathrm{~s}$, (c) $1.2 \mathrm{~s}$ and $3.0 \mathrm{~s}$ (d), after the application of the thermal excitation.

\subsection{Analysis in the frequency domain: Processing with PPT}

Using the superposition principle - which states that any wave, periodic or not, can be approximated by the sum of purely harmonic waves oscillating at difference frequencies - it is possible to extract and analyse the thermal waves oscillating at difference frequencies from the thermal response of the material after the application of a thermal pulse. 
This processing technique is known as Pulsed-Phase Thermography (PPT) [13] and consists in going from the time domain to the frequency domain, through the application of the pixel by pixel one-dimensional Fourier Transform to the thermal sequence.

Mathematically, the application of a thermal pulse is equivalent to a broadband frequency, having an energy distribution on each thermal wave components governed by the duration of the thermal pulse. The diffusion of heat from the surface into the material can be understood in terms of the propagation of these thermal waves into the solid away from the surface. The different frequency components being propagated travel at different speeds and suffer different amounts of attenuation. These components will also propagate according to the thermal diffusion length equation:

$$
\mu=\sqrt{\alpha / \pi \cdot f_{\text {lock-in }}}
$$

In Eq. (17), $\boldsymbol{\alpha}$ is the thermal diffusivity of the material (given by $\boldsymbol{k} / \boldsymbol{\rho} \boldsymbol{C}_{\boldsymbol{p}}$ ) and $\boldsymbol{f}_{\text {lock-in }}$ represents the frequency of the thermal wave. It can be noted from Eq. (17) that high frequency thermal waves propagate close to the surface and inversely, low frequency thermal waves propagate deeper.

Figure 4 shows different phasegrams at selected frequencies, obtained after the application of the Fourier Transform. It can be noted that deeper defects - such as the delaminations between the composite/metal interface - can be detected at higher frequencies (as showed in figure $4 a$ and $4 b$ ), while shallower defects will be clearly visible at lower frequencies.

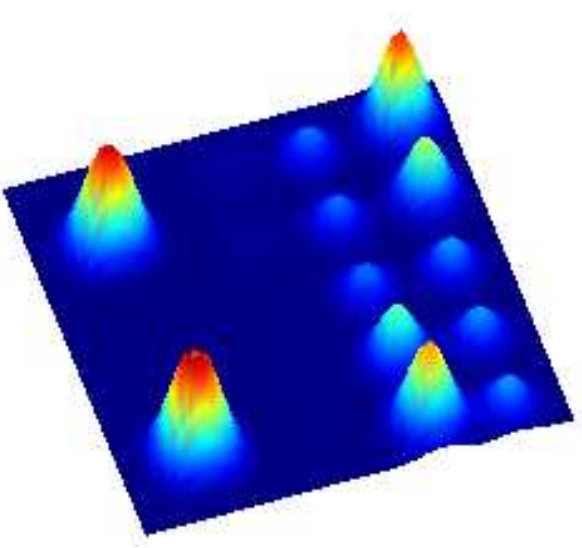

(a)

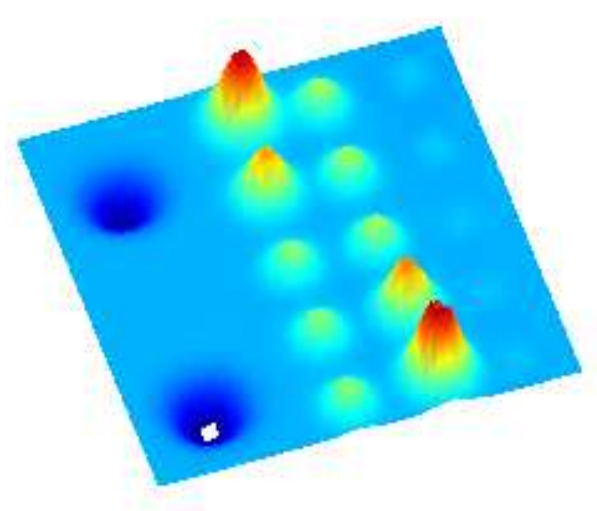

(c)
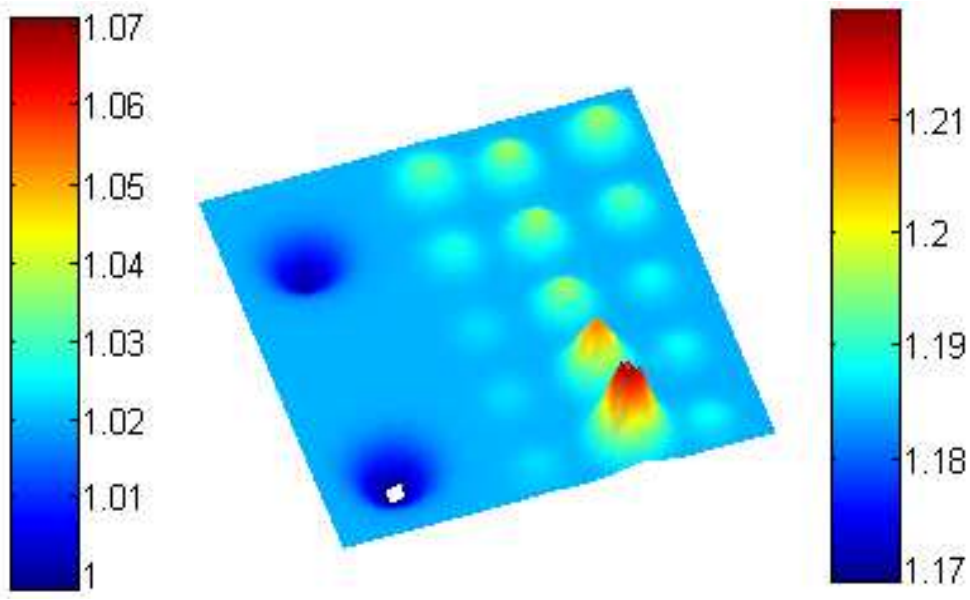

(b)
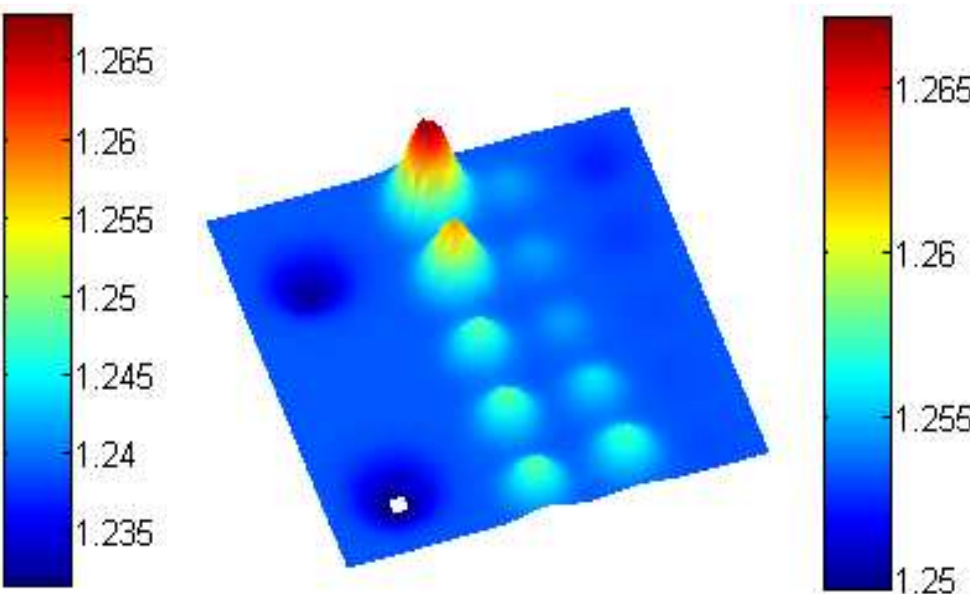

(d)

Fig. 4. Phasemaps obtained after the application of the Fourier Transform to the thermogram sequence.

Sampling frequency of $f_{s}=100 \mathrm{~Hz}$ and $N=600$. Selected frequencies are: (a) $0.167 \mathrm{~Hz}$; (b) $0.333 \mathrm{~Hz}$; (c) $1.17 \mathrm{~Hz}$; and (d) $1.67 \mathrm{~Hz}$. 


\section{Concluding remarks}

The results obtained by numerical simulation are consistent with the physical phenomena involved during the inspection by IRT. However, the improvement of the results greatly depends on the integration of real information related to the equipments used in a real TNDT, such as: characteristic associated to the infrared camera (time and frequency resolution), thermal radiation source (spectral and spatial distribution) and environment conditions under which the inspection is carried out. Future works consists in the adaption of those parameters to the computational tool developed; this will provide more reliable results and could be used as a prediction tool in real tests.

\section{REFERENCES}

[1] Maldague X. "Theory and Practice of Infrared Thermography for Nondestructive Testing". John Wiley and Sons, New York, 2001.

[2] Susa M., Castanedo-Ibarra C. And Maldague X. "Pulsed thermography applied on a complex structure sample: comparison and analysis of numerical and experimental results". IV Conferencia Panamericana de END, Buenos Aires, Argentina, 2007.

[3] KavianyM. "Principles of Heat Transfer in Porous Media". Springer, $2^{\text {nd }}$ edition, New York, 1995.

[4] Pan C.T. and Hocheng H. "The anisotropic heat-affected zone in the laser grooving of fiber-reinforced composite material". Journal of Materials Processing Technology, vol. 62, pp. 54-60, 1996.

[5] Rodriguez F. L. and Nicolau V. P."Inverse heat transfer approach for IR image reconstruction: Application to thermal non-destructive evaluation". Applied Thermal Engineering, vol. 33, pp. 109-118, 2012.

[6] Incropera F. and DeWitt D."Fundamentals of Heat and Mass Transfer". John Wiley \& Sons, Inc., $6^{\text {th }}$ edition, USA, 2007.

[7] Churchill S. W. and Chu H. S. "Correlating equations for laminar and turbulent free convection from vertical at plate". Int. J. Heat and Mass Transfer, vol. (18), pp. 1323-1329, 1975.

[8] MatLab: The Lenguage of Technical Computing. From the MathWorks ${ }^{\mathrm{TM}}$, Version 7.10.0.0499 (R2010a).

[9] Henderson J. H. and Wiecek T. E. "A mathematical model to predict the thermal response of decomposing, expanding polymer composites". Journal Comp. Materials, vol. 21, pp. 373-393, 1987.

[10] Callister W. "Materials Science and Engineering: An Introduction". John Wiley and Sons Ltd. $7^{\text {th }}$ edition, 2007.

[11] Mayr G., Dietermayr B., Hendorfer G. and Sekelja J. "Characterization of defects in curved CFRP samples using Pulsed Thermography and 3D finite element simulation”. $9^{\text {th }}$ International Conference on Quantitative Infrared Thermography, Krakow - Poland, 2008.

[12] Cielo P., Maldague X. and Krapez J.C. "Thermographic nondestructive evaluation: Data inversion procedures part ii: 2-d analysis and experimental results". Research in Nondestructive Evaluation, vol. 3, pp. 101-124, 1991.

[13] Maldague X., Galmiche F., Ziadi A. "Advances in pulsed phase thermography". Infrared Physics and Technology, vol. 43, pp. 175-181, 2002. 